\title{
Notification that New Names and New Combinations Have Appeared in Volume 44, No. 4, of the IJSB ${ }^{a}$
}

\begin{tabular}{|c|c|c|c|}
\hline Name: & Proposed as: & Authors: & $\begin{array}{l}\text { IJSB reference } \\
\text { description: }\end{array}$ \\
\hline Prevotella tannerae & sp. nov. & Moore et al. & 44(4):600 \\
\hline Prevotella enoeca & sp. nov. & Moore et al. & $44(4): 601$ \\
\hline Prevotella zoogleoformans & emend. & Moore et al. & $44(4): 602$ \\
\hline Methanolobus bombayensis & sp. nov. & Kadam et al. & 44(4):606 \\
\hline $\begin{array}{l}\text { Aeromicrobium fastidiosum (basonym Nocardioides } \\
\text { fastidiosa) }\end{array}$ & comb. nov. & Tamura and Yokota & 44(4):610 \\
\hline Desulfitobacterium & gen. nov. & Utkin et al. & 44(4):615 \\
\hline Desulfitobacterium dehalogenans & sp. nov. & Utkin et al. & $44(4): 615$ \\
\hline Rhodococcus luteus pro synon., Rhodococcus fascians & synon. & Klatte et al. & $44(4): 629$ \\
\hline Photobacterium histaminum & sp. nov. & Okuzumi et al. & $44(4): 634$ \\
\hline Porphyromonas gingivicanis & sp. nov. & Hirasawa and Takada & $44(4): 639$ \\
\hline Porphyromonas crevioricanis & sp. nov. & Hirasawa and Takada & 44(4):640 \\
\hline Streptococcus phocae & sp. nov. & Skaar et al. & 44(4):649 \\
\hline Bacillus laevolacticus & sp. nov., nom. rev. & Andersch et al. & 44(4):663 \\
\hline Rhodoplanes & gen. nov. & Hiraishi and Ueda & 44(4):671 \\
\hline Rhodoplanes roseus (basonym Rhodopseudomonas rosea) & comb. nov. & Hiraishi and Ueda & 44(4):671 \\
\hline Rhodoplanes elegans & sp. nov. & Hiraishi and Ueda & 44(4):672 \\
\hline Porphyromonas cangingivalis & sp. nov. & Collins et al. & $44(4): 676$ \\
\hline Porphyromonas cansulci & sp. nov. & Collins et al. & $44(4): 678$ \\
\hline Acholeplasma brassicae & sp. nov. & Tully et al. & 44(4):683 \\
\hline Acholeplasma palmae & sp. nov. & Tully et al. & $44(4): 683$ \\
\hline Mesoplasma pleciae & sp. nov. & Tully et al. & 44(4):690 \\
\hline Mesoplasma photuris & sp. nov. & Tully et al. & 44(4):691 \\
\hline Mesoplasma syrphidae & sp. nov. & Tully et al. & 44(4):691 \\
\hline Mesoplasma chauliocola & sp. nov. & Tully et al. & 44(4):691 \\
\hline Mesoplasma corruscae & sp. nov. & Tully et al. & 44(4):691 \\
\hline Mesoplasma grammopterae & sp. nov. & Tully et al. & 44(4):691 \\
\hline Mesoplasma coleopterae & sp. nov. & Tully et al. & 44(4):692 \\
\hline Mesoplasma tabanidae & sp. nov. & Tully et al. & 44(4):692 \\
\hline Sinorhizobium & emend. & De Lajudie et al. & 44(4):731 \\
\hline Sinorhizobium meliloti (basonym Rhizobium meliloti) & comb. nov. & De Lajudie et al. & 44(4):731 \\
\hline Sinorhizobium saheli & sp. nov. & De Lajudie et al. & 44(4):732 \\
\hline Sinorhizobium teranga & sp. nov. & De Lajudie et al. & $44(4): 732$ \\
\hline Sinorhizobium fredii & emend. & De Lajudie et al. & 44(4):732 \\
\hline Desulfuromusa & gen. nov. & Liesack and Finster & 44(4):756 \\
\hline Desulfuromusa kysingii & sp. nov. & Liesack and Finster & 44(4):757 \\
\hline Desulfuromusa bakii & sp. nov. & Liesack and Finster & 44(4):757 \\
\hline Desulfuromusa succinoxidans & sp. nov. & Liesack and Finster & 44(4):757 \\
\hline Actinopolyspora iraqiensis & sp. nov. & Ruan et al. & 44(4):760 \\
\hline Rhodococcus chubuensis pro synon., Gordona sputi & synon., emend. & Riegel et al. & 44(4):766 \\
\hline Gordona amarae (basonym Nocardia amarae) & comb. nov. & Klatte et al. & $44(4): 771$ \\
\hline Gordona aichiensis (basonym Rhodococcus aichiensis) & comb. nov. & Klatte et al. & 44(4):772 \\
\hline Halococcus salifodinae & sp. nov. & Denner et al. & 44(4):779 \\
\hline Haloincola saccharolytica & emend. & Cayol et al. & 44(4):809 \\
\hline Haloincola saccharolytica subsp. saccharolytica & & Cayol et al. & 44(4):810 \\
\hline Haloincola saccharolytica subsp. senegalensis & subsp. nov. & Cayol et al. & 44(4):810 \\
\hline Oxobacter & gen. nov. & Collins et al. & $44(4): 822$ \\
\hline Oxobacter pfennigii (basonym Clostridium pfennigii) & comb. nov. & Collins et al. & $44(4): 822$ \\
\hline Oxalophagus & gen. nov. & Collins et al. & 44(4):822 \\
\hline Oxalophagus oxalicus (basonym Clostridium oxalicum) & comb. nov. & Collins et al. & 44(4):822 \\
\hline Caloramator & gen. nov. & Collins et al. & 44(4):822 \\
\hline Caloramator fervidus (basonym Clostridium fervidus) & comb. nov. & Collins et al. & 44(4):822 \\
\hline Filifactor & gen. nov. & Collins et al. & 44(4):822 \\
\hline Filifactor villosus (basonym Clostridium villosum) & comb. nov. & Collins et al. & 44(4):822 \\
\hline Moorella & gen. nov. & Collins et al. & 44(4):822 \\
\hline $\begin{array}{l}\text { Moorella thermoacetica (basonym Clostridium } \\
\text { thermoaceticum) }\end{array}$ & comb. nov. & Collins et al. & $44(4): 824$ \\
\hline $\begin{array}{l}\text { Moorella thermoautotrophica (basonym Clostridium } \\
\text { thermoautotrophicum) }\end{array}$ & comb. nov. & Collins et al. & 44(4):824 \\
\hline Paenibacillus durum (basonym Clostridium durum) & comb. nov. & Collins et al. & 44(4):824 \\
\hline
\end{tabular}




\begin{tabular}{|c|c|c|c|}
\hline Name: & Proposed as: & Authors: & $\begin{array}{l}\text { IJSB reference } \\
\text { description: }\end{array}$ \\
\hline Eubacterium barkeri (basonym Clostridium barkeri) & comb. nov. & Collins et al. & $44(4): 824$ \\
\hline Thermoanaerobacter kivui (basonym Acetogenium kivui) & comb. nov. & Collins et al. & 44(4):824 \\
\hline $\begin{array}{l}\text { Thermoanaerobacter thermocopriae (basonym Clostridium } \\
\text { thermocopriae) }\end{array}$ & comb. nov. & Collins et al. & $44(4): 824$ \\
\hline $\begin{array}{l}\text { Thermoanaerobacterium thermosaccharolyticum (basonym } \\
\text { Clostridium thermosaccharolyticum) }\end{array}$ & comb. nov. & Collins et al. & $44(4): 824$ \\
\hline Chryseobacterium & gen. nov. & Vandamme et al. & 44(4):829 \\
\hline Chryseobacterium gleum (basonym Flavobacterium gleum) & comb. nov. & Vandamme et al. & $44(4): 830$ \\
\hline $\begin{array}{l}\text { Chryseobacterium balustinum (basonym Flavobacterium } \\
\text { balustinum) }\end{array}$ & comb. nov. & Vandamme et al. & 44(4):830 \\
\hline $\begin{array}{l}\text { Chryseobacterium indologenes (basonym Flavobacterium } \\
\text { indologenes) }\end{array}$ & comb. nov. & Vandamme et al. & 44(4):830 \\
\hline $\begin{array}{l}\text { Chryseobacterium indoltheticum (basonym Flavobacterium } \\
\text { indoltheticum) }\end{array}$ & comb. nov. & Vandamme et al. & $44(4): 830$ \\
\hline $\begin{array}{l}\text { Chryseobacterium meningosepticum (basonym } \\
\text { Flavobacterium meningosepticum) }\end{array}$ & comb. nov. & Vandamme et al. & 44(4):830 \\
\hline $\begin{array}{l}\text { Chryseobacterium scophthalmum (basonym Flavobacterium } \\
\text { scophthalmum) }\end{array}$ & comb. nov. & Vandamme et al. & 44(4):830 \\
\hline Bergeyella & gen. nov. & Vandamme et al. & 44(4):830 \\
\hline Bergeyella zoohelcum (basonym Weeksella zoohelcum) & comb. nov. & Vandamme et al. & 44(4):830 \\
\hline Empedobacter & gen. nov., nom.rev. & Vandamme et al. & 44(4):830 \\
\hline Empedobacter brevis (basonym Flavobacterium breve) & comb. nov. & Vandamme et al. & 44(4):830 \\
\hline
\end{tabular}

${ }^{a}$ This listing of names published in a previous issue of IJSB is provided as a service to bacteriology to assist in the recognition of new names and new descriptions. This procedure was proposed by the Judicial Commission [Minute II (ii), Int. J. Syst. Bacteriol. 41:185, 1991]. The names given herein have priority according to the issue of the IJSB in which they were published. 